\title{
MS25-P04 | DedicATEd ELECTRON SOURCE FOR SERIAL ELECTRON CRYSTALLOGRAPHY
}

Hogan-Lamarre, Pascal (University of Toronto, Toronto, CAN); Khazai, Nariman (University of Toronto, Toronto, CAN); Bücker, Robert (Max Planck Institute for the Structure and Dynamics of Matter, Hamburg, GER); Kassier, Günther (Max Planck Institute for the Structure and Dynamics of Matter, Hamburg, GER); Miller, R.J.Dwayne (University of Toronto, Toronto, CAN)

Work in electron-based macromolecular structure determination has grown in importance with the recent developments in rotation electron crystallography [1]. It was recently shown that serial electron crystallography (serialED) can be implemented within an electron microscope (TEM) to recover structures from nanocrystalline inorganic molecules [2]. This raises questions on the applicability of serialED to organic macromolecules. Such materials present challenges, notably in terms of dose sensitivity and beam quality. Moreover, TEMs have their own limitations: spatial constraints forbid large-travel high-precision stages or equipment for dynamics triggering.

We present the design of a dedicated electron beamline for serialED in development. A Schottky field emitter filtered by a $50-\mu \mathrm{m}$ aperture creates a highly-coherent beam. Sub- $\mu$ s pulses are generated through pulsing of the extraction potential and beam blanking. Properties of the beamline are explored through particle-tracking simulations based on realistic representations of the optics from finite-element methods. Macromolecular structures determination in a high-current regime is discussed: considering a fluence threshold of 5 e-/ $/ \AA^{2}$, simulations show that a repetition rate of the order of $100 \mathrm{~Hz}$ is achievable.

Data processing is explored in a proof-of-principle experiment within a TEM. Using softwares developed for $x$-ray diffraction, such as CrystFEL ${ }^{4}$ and $\mathrm{CCP} 4$, the structures of hen-egg lysozyme, and granulovirus are recovered at a resolution of $2 \AA$ and $1.8 \AA$, respectively.

[1] D.Shi et al., eLife, (2013).

[2] S.Smeets et al., J. Appl. Cryst 51, (2018).

[3] T.A.White et al., J. Appl. Cryst 45, (2012). 